\title{
Feasibility and benefit of long-dose stroke neurorehabilitation which produced and maintained balance and mobility gains throughout cancer surgery and radiation treatment
}

\author{
Catherine Boissoneault ${ }^{1}$, Anna Khanna ${ }^{1}$ and Janis J Daly ${ }^{1,2 *}$ \\ ${ }^{1}$ Department of Neurology, College of Medicine, University of Florida, Gainesville Florida \\ ${ }^{2}$ Brain Rehabilitation Research Center, North Florida/South Georgia VA Medical Center, Gainesville Florida
}

\begin{abstract}
Purpose: This case study provides quantitative evidence of the feasibility and benefit of a long-dose, 10-month neurorehabilitation program for a chronic stroke survivor who, in the midst of the neurorehabilitation program, underwent surgery and radiation treatment for breast cancer.

Methods: The patient was age 60 years, and 6 years post-stroke (left middle cerebral artery stroke), exhibiting impairments in balance, strength, and gait coordination, as well as deficits in functional mobility, and compromised quality of life. Her comprehensive mobility/fitness neurorehabilitation program included aerobics, strength and coordination training, and balance and gait coordination training. Treatment was 1.5 - 2 hours per day, 5 days/week for 6 months, followed by an additional 4 months of treatment 3 days/week. Outcome measures were acquired at entry into the neurorehabilitation program, and at months 2, 4, 6, 8 and 10. Measures included the following: modified Ashworth (mASH, spasticity); Fugl-Meyer (FM; isolated limb joint coordination); 6-minute walk test (6MWT; walking endurance); gait speed; Berg Balance Scale (BBS; static and dynamic balance); Timed Up and Go (TUG; mobility); 10 Meter Walk Test (10MWT; short distance, self-selected and fast walking speed); Functional Gait Assessment (FGA; dynamic balance and postural stability during gait); Functional Independence Measure (FIM; assessment of functional status and disability); Craig Handicap Assessment and Reporting Technique (CHART; quality of life assessment of how patients with disabilities function in the community and at home); 36-Item Short Form Survey (SF36; quality of life measure); and the Stroke Impact Scale (SIS; stroke-specific quality of life measure). Fatigue level was queried during and following radiation therapy.
\end{abstract}

Results: From baseline through month 4, the patient demonstrated improvements in most outcome measures. For example, she improved from 3 minutes of cycling endurance to 45 minutes, and balance improvement reached the threshold for functional independence. At month 5 , she was diagnosed with breast cancer and underwent a partial mastectomy. She underwent radiation treatment, which concluded in month 7. Prior, during, and after the cancer treatment, she continued participation in the neurorehabilitation program.

Following the partial mastectomy, she exhibited continued improvement in BBS, 10MWT-normal speed, FGA, CHART, SF-36, and SIS. Notably, the TUG continued to improve significantly by $4.73 \mathrm{sec}$. She showed some decline in values for the 6MWT, gait speed, and 10MWT- fast walking speed.

Four weeks following the end of the course of radiation therapy, most measures showed a small decline. But in contrast, by 12 weeks after the end of radiation (which coincided with 10 months of participation in the neurorehabilitation program), the following measures showed a revival in improvement: Fugl-Myer (limb coordination); 6MWT (walking endurance); gait speed; BBS (balance); FIM (functional tasks); subdomains of the CHART quality of life measure (physical independence, cognitive independence); SF-36 subdomain of energy/fatigue; and the SIS (subdomains of strength, memory and thinking, and mobility.

Conclusions: Years after stroke, intensive, long-dose neurorehabilitation can improve lower limb coordination, balance, strength, mobility, activities of daily living/ instrumental activities of daily living (ADL/IADL), overall function, and quality of life. It was feasible for a severely impaired stroke survivor to participate in an intensive neurorehabilitation program. It was feasible for a stroke survivor to participate in a neurorehabilitation program throughout surgical and radiation treatment for cancer. For a chronic stroke survivor, many aspects of impairment, function, and quality of life were maintained by neurorehabilitation throughout treatment for cancer.

\section{Introduction}

To our knowledge, there have been no studies published on the response to neurorehabilitation for the co-morbidities of chronic stroke and breast cancer. A 2018 statement from the American Heart Association [1] highlights the shared risk factors between breast cancer and cardiovascular disease, as well as the cardiotoxic effects of radiation and chemotherapy (including hypertension, arrhythmias, and thromboembolic disease) and the associated increased mortality risk due to cardiovascular disease in post-menopausal breast cancer survivors. However, the impact of cancer treatment on a chronic stroke survivor's neurorehabilitation response has not been explored. Therefore, this case study provides the treatment response to a longdose, 10-month neurorehabilitation program for a chronic stroke

${ }^{\star}$ Correspondence to: Janis J Daly, Department of Neurology, College of Medicine, University of Florida, Gainesville Florida, Tel: (352) 273-5550; Fax: (352) 273-5575; E-mail: janis.daly@neurology.ufl.edu

Received: April 25, 2018; Accepted: May 07, 2018; Published: May 09, 2018 
survivor with breast cancer receiving surgery and radiation during neurorehabilitation.

\section{Methods}

Ms. White (name changed for confidentiality) was age 60 years. She was 6 years post-stroke (left middle cerebral artery stroke) when she enrolled in the long-dose neurorehabilitation program. She exhibited impairments in balance, strength, and gait coordination, as well as deficits in functional mobility, and compromised quality of life. Her neurorehabilitation program included a comprehensive mobility and fitness training program, during which she received 1.5 - 2 hours of physical therapy per day in an outpatient clinic, 5 days/ week for 6 months, followed by an additional 4 months of treatment 3 days/week. The neurorehabilitation program included the following: moderate intensity aerobic training on a recumbent cycle for $45 \mathrm{~min}$ twice weekly, using methods established by Quaney, et al. [2]; strength and gait coordination training 3-5 days/week per established protocols $[3,4]$. Strength training included 1-3 days/week at 50-80\% 1RM, 6-12 reps per set, 1-4 sets per muscle group. Balance training was 2-3 days/ week, including a combination of Yang-style Tai Chi movements and conventional static and dynamic balance training on varying surfaces; and voluntary muscle activation re-training was integrated with coordination exercises and surface functional electrical stimulation [4].

During participation in this neurorehabilitation program, she was diagnosed with breast cancer (right breast; infiltrating ductal carcinoma (estrogen-receptor-positive, progesterone-receptor-positive, human epidermal growth factor receptor 2 positive, grade 2). She underwent a partial mastectomy procedure in month 5 of the neurorehabilitation program. She was administered $1 \mathrm{mg}$ /day Anastrozole and received a 4 -week course of radiation therapy, which concluded at the end of month 7 of her neurorehabilitation program.

Prior, during and after the cancer treatment, she continued participation in the neurorehabilitation program (10 months).

Outcome measures were acquired at entry into the neurorehabilitation program, and at months 2, 4, 6, 8 and 10. Measures included: modified Ashworth (mASH, spasticity); FuglMeyer (FM; isolated limb joint coordination); 6-minute walk test (6MWT; walking endurance); gait speed; Berg Balance Scale (BBS; static and dynamic balance); Timed Up and Go (TUG; mobility); 10 Meter Walk Test (10MWT; short distance, self-selected and. fast walking speed); Functional Gait Assessment (FGA; dynamic balance and postural stability during gait); Functional Independence Measure (FIM; assessment of functional status and disability); Craig Handicap Assessment and Reporting Technique (CHART; assessment of how patients with disabilities function in the community and at home); 36Item Short Form Survey (SF36; quality of life measure); and the Stroke Impact Scale (SIS; stroke-specific quality of life measure). Fatigue level was queried during and following radiation therapy.

\section{Results}

\section{Response to four months of the neurorehabilitation program (before cancer surgery and before radiation)}

Ms. White demonstrated improvements in most outcome measures (Table 1, columns A-C). Balance. Significant progress was made in balance. The BBS improved by 15 points (minimum detectable difference $(\mathrm{MDC})=4.13$ [5]. She achieved a score of $45 / 56$, which just achieves the level of 'functional independence' according to the 45point score threshold established by Doggan [6]. Joint coordination.
Her lower limb coordination improved 6 points (Fugl-Meyer MDC $=3.57$ [7]. Function. The Functional Gait Assessment improved 6 points (minimum detectable change $(\mathrm{MCD})=4.2$, Lin et al 2010). The mobility test, the TUG, improved 9.82sec (MDC $=3.16 \mathrm{sec}$; [7]). Aerobic capacity/fitness endurance progressed from initial capability of only 3 min continuous cycling to ability to cycling for $45 \mathrm{~min}$ at a moderate intensity level, $2 \mathrm{x} /$ week.

\section{After cancer surgery}

Following the partial mastectomy, she continued the neurorehabilitation program. She exhibited continued improvement in values for the BBS, 10MWT- normal speed, FGA, CHART, SF-36, and SIS (Table 1, column D, just after the surgery for cancer). Notably, the TUG continued to improve significantly by $4.73 \mathrm{sec}$. She showed some decline in values for gait speed including the 6MWT, gait speed, and 10MWT- fast walking speed. (Table 1, column D, after the surgery for cancer).

\section{After cancer radiation}

Four weeks after the end of course of radiation therapy (Table 1 , column E), most measures showed a small decline. In contrast to that, there was an improvement by 12 weeks after radiation (Table 1 , column F), which coincided with 10 months of participation in the neurorehabilitation program. Table 1, column $\mathrm{F}$ shows that the following measures improved from month 8 to month 10: Fugl-Myer (limb coordination), 6MWT (walking endurance), gait speed, BBS (balance), FIM (functional tasks), subdomains of the CHART quality of life measure (physical independence, cognitive independence), SF36 subdomain of energy/fatigue, and the SIS (subdomains of strength, memory and thinking, and mobility.

Notably, while participating in the neurorehabilitation program, Ms. White was able to either maintain or increase in many capabilities during and after the radiation treatment for cancer.

Those measures (Table 1, columns E and F) include the following: the FIM (functional tasks), the CHART quality of life subdomains (mobility and cognitive independence), the SF36 subdomain (life role limitations due to physical problems, energy/fatigue, emotional wellbeing), and SIS subdomains (strength, memory and thinking, emotion, activities of daily living (ADL) and instrumental activities of daily living (IADL), mobility, and participation in life roles).

Regarding fatigue level, prior to radiation, she reported zero fatigue at the time of query and slight fatigue at some point during the prior day of the query. After radiation, she reported fatigue slightly elevated above her own baseline, during weeks 1, 2, and 4 within the four-week radiation treatment, but no elevation of fatigue during the subsequent 8 weeks after the end of radiation treatment. In other words, any fatigue due to the four-week course of radiation treatment had resolved by the week after radiation and continued to be resolved throughout the subsequent 8 weeks.

Some weeks prior to Month 10 data collection (Table 1, column F), Ms. Jones had the flu and it was sufficiently debilitating as to require her to miss a week of the neurorehabilitation program. At the month 10 data acquisition the following measures showed a decline: 10MWT chosen speed, 10MTW fast speed, TUG (a measure of mobility speed), and selected SF36 subdomains (physical functioning, pain, and general healthy). This could have been due to having just had the flu. After radiation treatment, abnormal muscle tone increased (mASH, column E); by month 10 , abnormal muscle tone had decreased substantially, but not to baseline. 
Boissoneault C (2018) Feasibility and benefit of long-dose stroke neurorehabilitation which produced and maintained balance and mobility gains throughout cancer surgery and radiation treatment

Table 1. Data Collection Time Points During the Neurorehabilitation Program.

\begin{tabular}{|c|c|c|c|c|c|c|}
\hline Measure & A. Baseline & B. Month 2 & C. Month 4 & D. Month 6 & E. Month 8 & F. Month 10 \\
\hline \multicolumn{7}{|l|}{ Impairment Measures } \\
\hline $\begin{array}{l}\text { Fugl-Meyer coordination } \\
\text { (normal=34) }\end{array}$ & 16 & 22 & 22 & 22 & 20 & 21 \\
\hline $\begin{array}{l}\text { Modified Ashworth, } \\
\text { abnormal muscle tone } \\
(0=\text { normal })\end{array}$ & 4 & 6 & 7 & 6 & 12.5 & 9.5 \\
\hline \multicolumn{7}{|l|}{ Function Measures } \\
\hline 6MWT (feet walked) & 249 & 226 & 309 & 269 & 224 & 260 \\
\hline Gait Speed (m/sec) & 0.213 & 0.203 & 0.269 & 0.244 & 0.208 & 0.229 \\
\hline BBS normal=56) & 30 & 34 & 45 & 46 & 42 & 48 \\
\hline TUG (secs; less is better) & 51.45 & 51.77 & 41.63 & 36.9 & 49.2 & 52.15 \\
\hline $\begin{array}{l}\text { 10MWT self-chosen } \\
(\mathrm{m} / \mathrm{sec})\end{array}$ & 0.208 & 0.211 & 0.252 & 0.254 & 0.185 & 0.155 \\
\hline 10MWT fast $(\mathrm{m} / \mathrm{sec})$ & 0.231 & 0.234 & 0.303 & 0.272 & 0.218 & 0.188 \\
\hline FGA & 5 & 10 & 11 & 12 & 10 & 7 \\
\hline FIM (normal=126) & 109 & 110 & 113 & 113 & 113 & 114 \\
\hline \multicolumn{7}{|l|}{ Quality of life measure } \\
\hline CHART (total) (normal=600) & 385.7 & 450.8 & 489.3 & 513.8 & 513.95 & 501.17 \\
\hline Physical Independence & 59.2 & 92.8 & 91.3 & 92.8 & 90.95 & 96.467 \\
\hline $\begin{array}{l}\text { Cognitive } \\
\text { Independence }\end{array}$ & 56 & 70 & 76 & 76 & 94 & 100 \\
\hline Mobility & 100 & 97 & 100 & 100 & 100 & 99 \\
\hline Occupation & 48.5 & 66 & 72 & 95 & 79 & 55.7 \\
\hline Social Integration & 97 & 100 & 100 & 100 & 100 & 100 \\
\hline $\begin{array}{l}\text { Economic Self- } \\
\text { Sufficiency }\end{array}$ & 25 & 25 & 50 & 50 & 50 & 50 \\
\hline \multicolumn{7}{|l|}{ Mixed Measures } \\
\hline SF-36 (global avg) (normal=100) & 82.25 & 71.25 & 76.75 & 77.625 & 77.875 & 70.375 \\
\hline Physical Functioning & 25 & 30 & 40 & 40 & 35 & 30 \\
\hline Role Limitations due to Physical Problems & 100 & 75 & 100 & 75 & 100 & 100 \\
\hline Role Limitations due to Emotional Problems & 100 & 100 & 100 & 100 & 100 & 100 \\
\hline Energy/Fatigue & 75 & 60 & 65 & 55 & 75 & 80 \\
\hline Emotional Well- Being & 88 & 80 & 84 & 76 & 88 & 88 \\
\hline Social Functioning & 100 & 50 & 87.5 & 100 & 87.5 & 50 \\
\hline Pain & 100 & 100 & 67.5 & 100 & 57.5 & \\
\hline General Health & 70 & 75 & 70 & 75 & 80 & 70 \\
\hline SIS $($ total) $($ normal $=900)$ & 477.62 & 591.55 & 577.73 & 584.4 & 632.03 & 666.97 \\
\hline Strength & 25 & 37.5 & 37.5 & 25 & 43.75 & 50 \\
\hline Memory and Thinking & 75 & 85.71 & 78.57 & 78.57 & 85.71 & 92.86 \\
\hline Emotion & 83.33 & 77.78 & 83.33 & 83.33 & 94.44 & 91.67 \\
\hline Communication & 89.29 & 100 & 100 & 100 & 100 & 100 \\
\hline ADL/IADL & 57.5 & 75 & 80 & 77.5 & 80 & 80 \\
\hline Mobility & 50 & 80.56 & 83.33 & 75 & 75 & 80.56 \\
\hline Hand Function & 0 & 0 & 0 & 0 & 0 & 0 \\
\hline Participation/Role Function & 37.5 & 75 & 87.5 & 75 & 78.13 & 96.88 \\
\hline Stroke Recovery & 60 & 60 & 65 & 70 & 75 & 75 \\
\hline
\end{tabular}

\section{Discussion}

Stroke is a leading cause of disability worldwide [8] with associated high rates of physical inactivity. Natural history studies of chronic stroke survivors have demonstrated that residual deficits and strokeassociated comorbidities lead to negative health outcomes in the years following stroke [8]. Likewise, breast cancer treatment with surgery and radiation often result in persistent adverse effects, including pain, fatigue, lymphedema, muscle weakness, and decreased ability to perform ADLs [9]. This current case study contributes to the literature by demonstrating the advantages of continued neurorehabilitation for a stroke survivor during cancer surgery and radiation, and that an individual with chronic stroke can maintain a good deal of function and even make significant improvements in physical function and quality of life measures throughout a course of cancer treatment.
The primary side-effect of radiation therapy is fatigue. Radiationrelated fatigue typically impacts patients cumulatively over the course of several weeks, with a plateau in week 4 of radiation and recovery within 3 weeks of radiation treatment cessation [10]. A 2017 metaanalysis published by Lipsett et al. found a significant reduction in cancer-related fatigue when women with breast cancer participated in a combination of moderate intensity aerobic exercise and muscle strengthening programs during radiation treatment. Further, current clinical guidelines recommend participation in multi-modal exercise programs both during and after radiation therapy to optimize cancer outcomes and minimize treatment-related side effects [11]. These exercise recommendations are consistent with those utilized in our rehabilitation program for stroke survivors. A 2017 intervention study by Mostarda, et al. found that women undergoing treatment for 
breast cancer who participated in a 1-month aerobic and resistance exercise program significantly improved heart rate variability and cardiorespiratory impairments to levels that were comparable to healthy controls [12].

The stroke and cancer survivor case presented here tolerated the neurorehabilitation program well with little change in reported fatigue level both during and following her radiation therapy. The current case study provides evidence that a severely impaired stroke survivor can improve, even six years after the stroke. And it is feasible for a chronic stroke survivor with cancer to actively participate in an intensive, long-dose fitness/mobility exercise program. Most notably, this case illustrates that, even if a stroke survivor undergoes surgery and radiation treatment for cancer, a neurorehabilitation program can maintain many aspects of the individual's function and quality of life throughout the course of the cancer treatment. Importantly, neurorehabilitation can even support the continued improvement in function and quality of life for a stroke survivor during continued neurorehabilitation after recovery from cancer surgery and radiation. In the case of Ms. White, downwardly spiraling sequelae did not occur; rather, by month 10 of the neurorehabilitation program, most measures of function and quality of life were on an improvement trend, as had been occurring prior to the cancer treatment [13-14].

\section{Conclusions}

Years after stroke, intensive, long-dose neurorehabilitation can improve lower limb coordination, balance, strength, mobility, ADL/IADL's, overall function, and quality of life. It was feasible for a severely impaired stroke survivor to participate in an intensive neurorehabilitation program. It was feasible for a stroke survivor to participate in a neurorehabilitation program throughout surgical and radiation treatment for cancer. For a chronic stroke survivor, many aspects of impairment, function, and quality of life were maintained by neurorehabilitation throughout treatment for cancer.

\section{Acknowledgment of funding}

McKnight Brain Institute and College of Medicine, University of Florida, Gainesville, Florida, USA (grant number 00114654; study Principle Investigator, Dr. J Daly).

\section{References}

1. Mehta LS, Watson KE, Barac A, Beckie TM, Bittner V, et al. (2018) Cardiovascular disease and breast cancer: where these entities intersect: a scientific statement from the American Heart Association. Circulation 137: e30-e66.

2. Quaney BM, Boyd LA, McDowd JM, Zahner LH, He J, et al. (2009) Aerobic exercise improves cognition and motor function poststroke. Neurorehabil Neural Repair 23: 879-885.

3. Daly JJ, Zimbelman J, Roenigk KL, McCabe JP, Rogers JM, et al. (2011) Recovery of coordinated gait: randomized controlled stroke trial of functional electrical stimulation (FES) versus no FES, with weight-supported treadmill and over-ground training. Neurorehabil Neural Repair 25: 588-596.

4. Daly JJ, McCabe JP, Gansen J, Rogers J, Butler K, et al. (2012) Gait Coordination Protocol for recovery of coordinated gait, function, and quality of life following stroke. JRDD 49: xix-xix.

5. Flansbjer UB, Blom J, Brogårdh C (2012) The reproducibility of Berg Balance Scale and the Single-leg Stance in chronic stroke and the relationship between the two tests. PMR 4: 165-170. [Crossref]

6. Doggan A, MengüllüoGGlu M, Özgirgin N (2011) Evaluation of the effect of anklefoot orthosis use on balance and mobility in hemiparetic stroke patients. Disabil Rehabil 33: 1433-1439.

7. Hiengkaew V, Jitaree K, Chaiyawat P (2012) Minimal detectable changes of the Berg Balance Scale, Fugl-Meyer Assessment Scale, Timed "Up \& Go" Test, gait speeds, and 2-minute walk test in individuals with chronic stroke with different degrees of ankle plantarflexor tone. Archives of physical medicine and rehabilitation 93: 1201-1208.

8. Godwin KM, Ostwald SK, Cron SG, Wasserman J (2013) Long-term health related quality of life of survivors of stroke and their spousal caregivers. J Neurosci Nurs 45: 147.

9. Santa Mina D, Brahmbhatt P, Lopez C, Baima J, Gillis C, et al. (2017) The case for prehabilitation prior to breast cancer treatment. $P M R$ 9: S305-S316.

10. Greenberg DB, Sawicka J, Eisenthal S, Ross D (1992) Fatigue syndrome due to localized radiation. J Pain Symptom Manage 7: 38-45.

11. Hayes SC, Spence RR, Galvão DA, Newton RU (2009) Australian Association for Exercise and Sport Science position stand: optimising cancer outcomes through exercise. J Sci Med Sport 12: 428-434.

12. Mostarda C, Castro-Filha J, Reis AD, Sevílio Jr M, Dias CJ, et al. (2017) Shortterm combined exercise training improves cardiorespiratory fitness and autonomic modulation in cancer patients receiving adjuvant therapy. J Exerc Rehabil 13: 599.

13. Lin JH, Hsu MJ, Hsu HW, Wu HC, Hsieh CL (2010) Psychometric comparisons of 3 functional ambulation measures for patients with stroke. Stroke 41: 2021-2025.

14. Lipsett A, Barrett S, Haruna F, Mustian K, O'Donovan A (2017) The impact of exercise during adjuvant radiotherapy for breast cancer on fatigue and quality of life: A systematic review and meta-analysis. The Breast 32: 144-155.

Copyright: (C2018 Boissoneault C. This is an open-access article distributed under the terms of the Creative Commons Attribution License, which permits unrestricted use, distribution, and reproduction in any medium, provided the original author and source are credited. 